OPEN ACCESS

Edited by: Ashok Mysore, St. John's Medical College Hospital,

India

Reviewed by: Marco Grados, Johns Hopkins University School of Medicine, USA

Preeti Jacob,

National Institute of Mental Health and Neurosciences, India

${ }^{*}$ Correspondence: Akemi Tomoda atomoda@u-fukui.ac.jp

Specialty section: This article was submitted to Child and Neurodevelopmental Psychiatry, a section of the journal Frontiers in Psychiatry

Received: 02 July 2015 Accepted: 26 November 2015 Published: 11 December 2015

Citation:

Mizushima SG, Fujisawa TX, Takiguchi S, Kumazaki H, Tanaka S and Tomoda A (2015) Effect of the Nature of Subsequent Environment on Oxytocin and Cortisol Secretion in Maltreated Children.

Front. Psychiatry 6:173. doi: 10.3389/fpsyt.2015.00173

\section{Effect of the Nature of Subsequent Environment on Oxytocin and Cortisol Secretion in Maltreated Children}

\author{
Sakae G. Mizushima ${ }^{1,2}$, Takashi X. Fujisawa ${ }^{1,2}$, Shinichiro Takiguchi' ${ }^{2}$, Hirokazu Kumazaki ${ }^{1,2}$ \\ Shiho Tanaka ${ }^{2}$ and Akemi Tomoda ${ }^{1,2 *}$ \\ 'Division of Developmental Higher Brain Functions, United Graduate School of Child Development, Osaka University, \\ Kanazawa University, Hamamatsu University School of Medicine, Chiba University and University of Fukui, Fukui, Japan, \\ ${ }^{2}$ Research Center for Child Mental Development, University of Fukui, Fukui, Japan
}

Childhood maltreatment (CM), including abuse and neglect, is a crucial factor that distorts child development. $\mathrm{CM}$ is associated with alterations in numerous brain regions, and may be associated with hormonal dysregulation. This study aimed to investigate differences in secretion patterns of cortisol (CT) and oxytocin (OT) among children who experienced $\mathrm{CM}$, children living in residential care facilities and in unstable environments. Among 38 maltreated children, 23 (mean age $=12.2$ years, SD $=3.0$ ) were categorized as "Settled" and 15 (mean age $=13.1$ years, SD = 2.2) as "Unsettled." Twenty-six age- and gender-matched (mean age $=12.6$ years, $S D=2.1$ ), typically developing (TD) children were also included. Clinical and psychological assessments, including IQ and trauma evaluations, were conducted for all participants. Age, gender, and full-scale IQ were used as covariates in hormone analysis. Two saliva samples were collected, one on awakening and the other at bedtime. There were significant differences in the awakening CT levels of the "Unsettled" group, and in bedtime OT levels in the "Settled" group as compared with TD children, and between CM groups. Furthermore, there was a significant difference in trauma-symptomatic depression scores between the "Settled" and "Unsettled" CM group. These results suggest that CT diurnal secretions tend to be reactive to current stress rather than previous experience. OT diurnal secretions are presumably hyper-regulated for coping with the environment to survive and thrive. By measuring salivary CT/OT diurnal patterns, hormonal dysregulation of CM children living in "Settled" environments and "Unsettled" environments was indicated.

Keywords: child maltreatment, cortisol, oxytocin, residential care facility, hormones

\section{INTRODUCTION}

Childhood and adolescence is a time of remarkable change in physical and psychological development, brain structure, and neuroendocrine function. Although the process is guided by genetic factors, the final form is determined by life experience and the living environment. From birth, parent-infant interaction modulates fundamental brain processes $(1,2)$, whereas more complex psychological development occurs in socializing with peers (3). 
Adverse experience in the form of childhood maltreatment (CM) has been reported to bring several lifelong difficulties (4). For example, CM distorts attachment to, and sense of intimacy toward, "significant others." Therefore, many maltreated children suffer from interpersonal communication problems throughout their lives. Thus, exposure to $\mathrm{CM}$ is acknowledged to impact mental health (5), causing repercussions in social environments, including parents, family, and local communities.

Childhood Maltreatment is also associated with alterations in the size $(6,7)$ and functional activity of a variety of brain regions (8), and with hormonal dysregulation (9). Prior studies (10-14) have demonstrated that CM leads to abnormal reactivity of hormones, such as cortisol (CT) and oxytocin (OT). These effects extend into adulthood (10-12) as well as influencing childhood $(13,14)$. Thus, early life stress is crucial in exacerbating mental health issues in the general population, as well as in psychiatric populations.

Cortisol is released in response to stress via the hypothalamic-pituitary-adrenal (HPA) axis. The secretion is regulated by capturing negative feedback relating to stress. CT levels are relatively high in the morning and decrease by evening $(15,16)$. Reportedly, children's CT reactivity to stress is an important mediator of depression risk $(17,18)$. In addition, several studies of children with adverse childhood experiences (ACEs), such as institutionalized children in Romania $(19,20)$ and foster children $(13,21,22)$, have indicated atypical "blunted" CT diurnal secretions. Prior CT studies related to CM have shown high CT levels in maltreated children with PTSD $(23,24)$ and elevated morning CT levels (25) in foster children who experienced severe emotional maltreatment. Although there exist inconsistencies regarding CT levels in maltreated children, previous studies of CM and depression in childhood suggest HPA axis dysregulation as a result of CT stress reactions.

The neuropeptide OT is secreted from the posterior pituitary gland, and has physiological functions during labor and lactation. There is a growing body of evidence that OT plays an important role in regulating social behavior in diverse species $(26,27)$. In humans, many studies have suggested that OT modulates mother-infant bonding, and OT receptors are involved in social behavior, including reproductive and maternal behaviors, as well as affiliation and attachment (28-31). It has been suggested that OT plays an important role in attachment formation or bonding with a "primary care-giver," and individuals who have experienced that $\mathrm{CM}$ have atypical OT secretion patterns (32).

According to a Japanese government child welfare report in 2015 (33), more than 29000 children are institutionalized, such as in residential care homes, and $60 \%$ of those children have experienced CM. Before placing a child in institutionalized care, the child guidance center separately conducts several assessments of the children and their families; children are removed from their homes and placed with other children in temporary custody during the assessment. After the assessment, less than $30 \%$ of the children are placed in institutions [for more information about the institutionalized care system in Japan, see Suzuki and Tomoda (34)]. The remaining $67 \%$ of children return to their biological family homes.
Although the association between stressful events in childhood and abnormal hormonal levels are well established, few studies have examined the influence of CM on neuroendocrine function and pathways. Thus, developmental psychopathologists are highly interested in elucidating children's HPA axis dysregulation, indexed via CT and/or OT reactivity, with two distinct literatures $(13,35)$ emerging on role of the environment following CM experiences, especially regarding differences in living environment.

This study explored the effect of the subsequent environment (i.e., stable or unstable placement) on salivary CT/OT secretion patterns in maltreated children. We hypothesized that differences in neuroendocrine function would be present when comparing those children in stable versus unstable environments. We divided the maltreated children into two groups according to their living environments, and analyzed salivary CT/OT secretion patterns as well as their association with psychological scales. Typically developing (TD) children acted as control subjects in our study.

\section{MATERIALS AND METHODS}

\section{Ethics Statement}

The study protocol approved by the Ethics Committee of the University of Fukui (Assurance No. FU25-149). All participants' parent(s) and the caregivers who took custody of the child gave written informed consent for participation in the study. The experimental protocol was conducted in accordance with the Declaration of Helsinki.

\section{Participants}

This study involved 38 children who experienced CM, consisting of 19 boys and 19 girls. The children were subcategorized as "Unsettled" or "Settled." The "Unsettled" group was defined as children who have been living with their biological parent(s), and also children living in residential childcare homes where the length of stay was less than 1 year. The reason for adopting the stay length of less than 1 year was based on our unpublished data from our previous study (34): we found that depressive mental state within the first year of institutionalization was significantly higher than that from the second year and more. Fifteen children (six boys and nine girls; mean age 13.1 years, SD 2.1) were categorized as "Unsettled." The "Settled" group was defined as children who were settled in residential care homes instead of their original family homes. The duration within the settled environment ranged from a minimum of 1 year to a maximum of the child's current age (the longest duration was 15 years). Twenty-three children (13 boys and 10 girls; mean age, 12.2 years, $\mathrm{SD}=2.9$ ) were categorized as "Settled." Furthermore, 26 TD children (12 boys and 14 girls; mean age, 12.7 years, $\mathrm{SD}=2.2$ ) were included as healthy controls. The clinical patients who had experienced $\mathrm{CM}$ were recruited following their referral to the Department of Child and Adolescent Psychological Medicine at the University of Fukui Hospital via the local authority's child guidance centers, other children were recruited to the CM group after their parents brought them to the hospital for medical attention related to difficulties in child rearing. Almost all CM group children were 
primarily screened regarding their ACE by the child guidance center before referral. The types of abuse experienced in the CM group were as follows: neglect (62\%), physical abuse (35\%), emotional abuse (16\%), and sexual abuse (14\%). In the public child welfare system in Japan, detailed duration of maltreatment experiences by a child is protected, along with the identity of the abuser, socioeconomic status, and home environment, so we could not access this information even though our purpose is academic, and the children's identities would be protected.

The healthy age- and gender-matched TD controls were recruited from the general community in Fukui prefecture. They were recruited from the community through advertisements in a local newspaper and on our website homepage.

All participants' race/ethnicity was Japanese. Participants were primarily screened by the MINI-International Neuropsychiatric Interview for children and adolescents (MINI-KID: Japanese version) $(36,37)$. None of the TD children had any history of Diagnostic and Statistical Manual of Mental Disorders, Fourth Edition, Text Revision (DSM-IV-TR) (38) Axis I Disorders, including developmental disorders, or any history of any form of abuse or drug abuse, head injury, any physical or abnormal developmental milestones, or fetal drug exposure. The intellectual ability of the participants was assessed by the Wechsler Intelligence Scale for Children-Fourth Edition (WISC-IV) (39).

\section{Assessment Measures}

Previous studies have suggested that maltreated children have symptoms in common with neurodevelopmental disorders, such as attention-deficit hyperactivity disorder (ADHD) (40) and autism spectrum disorder (ASD) (41). In order to evaluate ADHD traits and ASD traits, the Japanese version of the ADHD Rating Scale-IV (42) and the Autism-Spectrum Quotientchildren's version (AQ) (43) were administered. The ADHD-RS (44) scale screens for children's behavior during their routine activities. It consists of 18 questions, which are divided into two subcategories, consisting of nine inattention items and nine hyperactivity-impulsivity items. The AQ measures autistic traits of children (45) via 50 questions that are rated by the child's parent or caretaker.

To assess behavioral problems, parents or caregivers completed the Strengths and Difficulties Questionnaire (SDQ) (46) which consists of 25 questions, split into 5 categories that assess children's internalized (emotional and peer relationships) and externalized (conduct and hyperactivity) problems ("difficulties"). The prosocial score is excluded from the total score, because the former is categorized as a positive quality of the individual. SDQ data were obtained from the children and via their parent or caretaker as well. Depressive mental state was assessed via the Depression Self-Rating Scale for Children (DSRS-C: Japanese version) (47), which contains 18 questions that probe emotional and physical reactions associated with the current mood of children.

Traumatic experiences related to CM were evaluated by the UCLA PTSD Index for DSM-IV (UPID) (48), the Impact of Event Scale Revised (IES-R) $(49,50)$, and the trauma symptom checklist for children (TSCC) (51). UCLA PTSD Index consists of 47 questions, which follow the diagnostic criteria for PTSD from the DSM-IV (38). The evaluation usually takes 20-30 min and directly stimulates children; therefore, they are able to disclose their experiences naturally. IES-R is also structured by the diagnostic criteria of PTSD from the DSM-IV. This self-rating consists of 22 questions that ask how the individual's thoughts and emotional state during the past week are influenced by previous trauma. TSCC consists of 54 questions, split into the six subcategories Anxiety, Depression, Anger, Post-traumatic Stress (PTS), Dissociation, and Sexual Concerns. These scales were applied during the structured interview by a licensed clinical psychologist (first author).

\section{Saliva Sampling}

Awakening and bedtime saliva CT/OT data were obtained using Salivettes ${ }^{\circledR}$ (Sarstedt, Rommelsdorft Corp., Germany). The awakening saliva sample was taken on waking, before breakfast (within $1 \mathrm{~h}$ of awakening). The bedtime saliva sample was taken before bedtime. The saliva sampling procedures were the same as those in our previous study (52). Before using Salivettes, instructions were given to all participants. Saliva samples were frozen and stored at $-80^{\circ} \mathrm{C}$ in the laboratory. Salivary CT and OT levels were measured by enzyme-linked immune sorbent assay (ELISA). Before assay, each saliva sample was restored to room temperature. The assay was conducted using an all-in-one CT kit (Salimetrics ${ }^{\mathrm{TM}}$ ). These protocols are based on prior studies of children (53-55). The saliva samples were also used for OT analysis. After completing CT data analysis, the samples were lyophilized overnight and kept at $-20^{\circ} \mathrm{C}$ to concentrate them two to four times. Afterwards, the dry samples were reconstructed in the assay buffer immediately before analysis, using an OT enzyme immunoassay commercial kit (Assay Designs Inc., Ann Arbor, MI, USA). The CT sample assay was duplicated, whereas the OT assay was executed in singlicate. The data were calculated using a SpectraMax ${ }^{\circledR}$ (Molecular Device, Sunnyvale Corp., CA, USA) micro plate reader, according to relevant standard curves. Average intra- and inter-assay coefficients of variation were 9.6 and $8.6 \%$, respectively.

\section{Statistical Procedure}

Three different groups were defined, in order to determine characteristic features of maltreated children ("Unsettled," "Settled," and TD). Differences among the three groups in the clinical variables were assessed using one-way analysis of covariance (ANCOVA). Analysis of biological data (i.e., salivary CT and OT) involved repeated-measures two-way ANCOVA. Statistical analyses were conducted using IBM SPSS Statistics 21.0 software (SPSS Inc., Chicago, IL). All $p$-values were two-tailed; $p$-values less than.05 were considered significant.

\section{RESULTS}

\section{Demographic Data and Clinical Assessments}

Table 1 shows the demographic and clinical characteristics of each group. The three groups were well matched in gender and age. ANOVA revealed that both CM groups ("Settled" and "Unsettled") exhibited significantly lower full-scale IQ 
TABLE 1 | Demographic data and clinical ratings of maltreated children (unsettled and settled) and typically developing children.

\begin{tabular}{|c|c|c|c|c|c|}
\hline Ratings & Unsettled & Settled & TD & Statistics & $p$-value \\
\hline Number & 15 & 23 & 26 & - & - \\
\hline Gender(M:F) & $6: 9$ & $13: 10$ & $12: 14$ & - & - \\
\hline Age (mean $\pm \mathrm{SD})$ & $13.1 \pm 2.2$ & $12.2 \pm 3.0$ & $12.1 \pm 2.1$ & $F_{2,61}=0.60$ & ns \\
\hline FSIQ & $91.00 \pm 13.3^{b}$ & $91.87 \pm 9.6^{b}$ & $102.23 \pm 9.0^{\mathrm{a}}$ & $F_{2,61}=8.26$ & $<0.0006$ \\
\hline ADHD-RS ${ }^{d}$ & $12.87 \pm 10.6^{a}$ & $10.83 \pm 9.8^{a}$ & $2.50 \pm 2.4^{b}$ & $F_{2,60}=6.78$ & $<0.0022$ \\
\hline $\mathrm{AQ}^{\mathrm{a}}$ & $19.80 \pm 5.7$ & $20.22 \pm 8.9$ & $13.50 \pm 5.7$ & $F_{2,60}=2.52$ & $\mathrm{~ns}$ \\
\hline SDQ (self-rating) ${ }^{d}$ & $17.00 \pm 6.9^{a}$ & $15.70 \pm 7.3^{\mathrm{a}}$ & $7.50 \pm 4.8^{b}$ & $F_{2,60}=10.77$ & $<0.0001$ \\
\hline SDQ (parent-rating) ${ }^{d}$ & $14.00 \pm 4.0^{\mathrm{a}}$ & $13.70 \pm 9.0^{\mathrm{a}}$ & $6.04 \pm 3.9^{b}$ & $F_{2,60}=7.48$ & $<0.0013$ \\
\hline DSRS-C ${ }^{d}$ & $14.00 \pm 7.3^{a}$ & $10.65 \pm 6.7^{b}$ & $6.96 \pm 4.7^{b}$ & $F_{2,60}=5.97$ & $<0.0043$ \\
\hline IES-R ${ }^{d}$ & $25.47 \pm 25.5^{a}$ & $16.30 \pm 14.9^{a}$ & $2.65 \pm 5.0^{b}$ & $F_{2,60}=11.15$ & $<0.0001$ \\
\hline TSCC-anxiety ${ }^{d}$ & $54.07 \pm 19.4^{\mathrm{a}}$ & $48.13 \pm 13.8^{a}$ & $39.42 \pm 5.7^{b}$ & $F_{2,60}=6.35$ & $<0.0031$ \\
\hline TSCC-depression ${ }^{d}$ & $52.87 \pm 17.1^{a}$ & $43.04 \pm 9.8^{b}$ & $37.19 \pm 2.5^{c}$ & $F_{2,60}=13.24$ & $<0.0000$ \\
\hline TSCC-anger $^{d}$ & $48.80 \pm 13.5^{a}$ & $43.65 \pm 7.1^{\mathrm{a}}$ & $36.81 \pm 2.5^{b}$ & $F_{2,60}=9.67$ & $<0.0002$ \\
\hline TSCC-post-traumatic stress ${ }^{d}$ & $50.60 \pm 14.7^{a}$ & $44.74 \pm 8.4^{\mathrm{a}}$ & $37.58 \pm 3.9^{b}$ & $F_{2,60}=10.99$ & $<0.0001$ \\
\hline TSCC-dissociation $^{d}$ & $48.47 \pm 13.4^{a}$ & $47.26 \pm 11.3^{a}$ & $37.50 \pm 2.5^{b}$ & $F_{2,60}=11.09$ & $<0.0001$ \\
\hline TSCC-sexual concerns ${ }^{d}$ & $41.47 \pm 3.9$ & $44.04 \pm 9.7$ & $38.85 \pm 2.3$ & $F_{2,60}=1.80$ & ns \\
\hline
\end{tabular}

FSIQ, full-scale IQ; ADHD-RS, attention-deficit hyperactivity disorder rating scale; $A Q$, autism-spectrum quotient-children's version; SDQ, strength and difficulties questionnaire; DSRSC, depression self-rating scale for children (Birleson); IES- $R$, impact of event scale revised; TSCC, trauma symptom checklist for children. $\left.{ }^{a}\right\rangle^{b}(p<0.05)$, ${ }^{a}>{ }^{c}(p<0.05)$, ${ }^{b}>{ }^{c}(p<0.05),{ }^{d}$ Covariate items: FSIQ.

(FSIQ) scores than the TD group $[F(2,61)=8.22, p<0.0006]$. For the clinical characteristics analysis among groups, FSIQ was adjusted using ANCOVA. Both CM groups ("Settled" and "Unsettled") showed significantly higher scores than the TD group on ADHD-RS $[F(2,60)=6.78, p=0.0022]$, SDQ [Self-rating: $F(2,60)=10.77, p<0.0001$, Parental-rating: $F(2,60)=7.48, p=0.0013]$. However, for the DSRS-C, the "Unsettled" group scored significantly higher than the "Settled" group and TD children $[F(2,60)=5.97, p=0.0043]$. There were no significant differences in AQ scores $[F(2,60)=2.52$, $p=0.0893]$. These results suggest that $\mathrm{CM}$ group children ("Settled" and "Unsettled") suffered from more behavioral and mood-related problems than the TD children, whereas the "Settled" CM group reported less severe difficulties than the "Unsettled" CM group.

For the trauma evaluation, according to the UPID (48), of the 38 subjects in the CM group, 3 met the full diagnostic criteria for PTSD, 6 were categorized as having partial PTSD, and 9 had a traumatic symptom above the UPID cut-off. Overall, 18 of 38 (47\%) CM subjects had relatively mild to moderate PTSD symptoms. Both CM groups showed significantly higher scores than the TD group on the IES-R $[F(2,60)=11.15 p<0.0001]$ and most of TSCC sub-scales [Anxiety: $F(2,60)=6.35, p=0.0031$; Anger: $F(2,60)=9.67, p<0.0002$; PTS: $F(2,60)=10.99$, $p<0.0001$; Dissociation: $F(2,60)=11.09, p<0.0001]$. However, the "Unsettled" group generated significantly higher scores for TSCC-depression than the "Settled" group and TD group [F (82, $60)=13.24, p<0.00001]$. This result suggests that "Unsettled" children tended to be more depressive than "Settled" CM and TD group children. No significant differences were found in the number of exposures to different types of maltreatment in the CM children [neglect; $F(1,36)=2.97, p=0.093$, physical abuse $F(1,36)=0.52, p=0.477$, psychological abuse $F(1,36)=0.32$. $p=0.578$, sexual abuse $F(1,36)=1.53, p=0.224]$.

\section{Salivary Cortisol and Oxytocin}

We, first, explored differences between the CM and TD groups in salivary levels of CT and OT, by using ANCOVA with covariates consisting of age, gender, and FSIQ, which revealed no significant differences (awakening CT, $p=0.079$, bedtime CT, $p=0.333$; awakening OT, $p=0.119$, bedtime OT, $p=0.213$ ). Second, in order to examine differences in salivary levels of CT and OT between "Unsettled," "Settled," and TD groups, each salivary level was compared using repeated-measures two-way ANCOVA with covariates consisting of age, gender, and FSIQ; secretion slope (awakening and bedtime) as a within-participants factor, and environmental category ("Unsettled," "Settled," and TD) as a between-participants factor. For CT levels (Figure 1A), we found a main effect of the time of day $[F(2,58)=5.18, p=0.008]$. This indicates that the average CT level was significantly higher on awakening than at bedtime. The main effect of environmental category (i.e., CM influence) was not significant, however, the interaction effect with time of day was significant $[F(2,58)=5.18, p=0.008]$, indicating that the "Unsettled" environment effect was greater on awakening than at bedtime. For OT levels (Figure 1B), there was a significant difference in the bedtime OT level between CM groups ["Settled" and "Unsettled"; $F$ $(2,58)=4.00, p=0.05]$. This result indicates that "Settled" children's OT secretions markedly increased from awakening to bedtime.

Additional analyses were conducted to determine whether there were between-group differences in diurnal patterns of CT and OT secretion. For CT and OT secretions, the area under the curve (AUC) and diurnal slope (change in levels from awakening to the bedtime) were calculated, and group-wise effects investigated using between-participants one-way ANOVAs. For the AUC, there was no significant difference in CT and OT hormone levels among the groups. However, a significant difference in the CT slopes was found $[F(2,58)=4.16, p=0.02]$, as shown in Table 2, whereas the OT slopes did not differ among groups $[F(2,58)=1, p=0.374]$. Post hoc analysis using Bonferroni 
correction indicated that the decline in CT level from awakening to bedtime was significantly larger in the "Unsettled" group than the TD children $[F(2,58)=4.16, p=0.019]$. This result indicates that the "Unsettled" children's CT secretion levels dropped sharply compared to the other groups (Figure 1A). There were no significant correlations between TSCC-depression scores and salivary CT or OT levels, adjusting FSIQ as a covariate $(N=64$; CT awakening: $r=0.087, p=0.501$; CT bedtime: $r=0.084$, $p=0.518$; OT awakening: $r=-0.101, p=0.433$; OT bedtime: $r=-0.039, p=0.764)$.
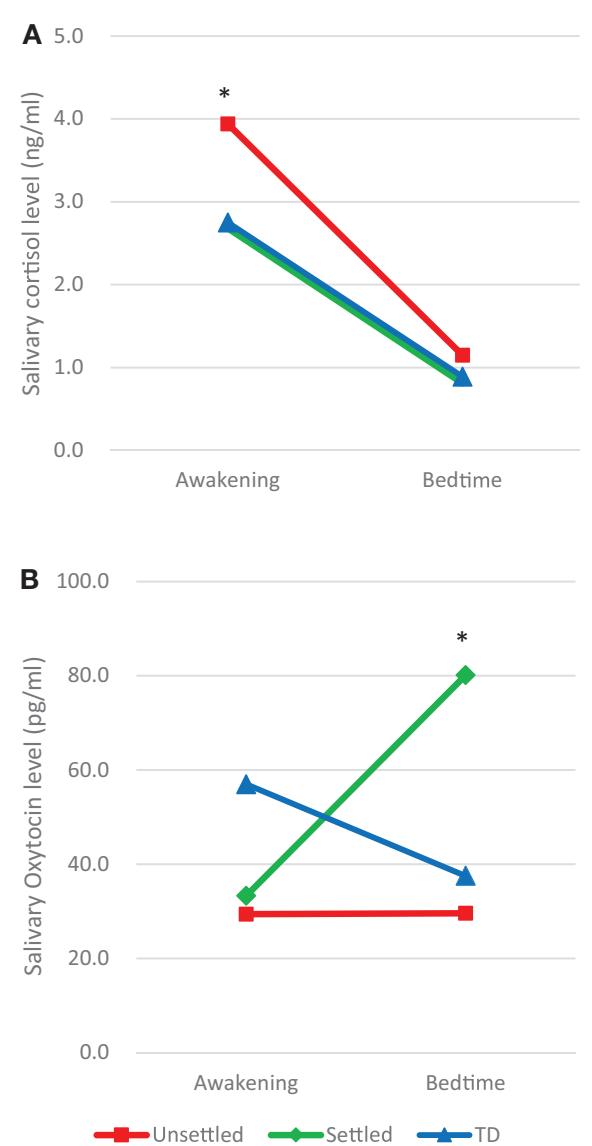

$*=p<.05$

FIGURE 1 | Diurnal secretion of salivary hormones in maltreated children (unsettled and settled) and typically developing children: (A) Cortisol and (B) Oxytocin.

\section{DISCUSSION}

The present study demonstrated distinct patterns of salivary levels of CT and OT secretions among maltreated children living in different environments ("Unsettled" and "Settled"), and TD children. There were significantly higher levels of awakening CT in the "Unsettled" group compared with the "Settled" group and TD group. Similarly, the "Unsettled" group had significantly higher DSRS-C scores than TD group, and higher TSCCdepression scores than the "Settled" and TD groups. These results suggest that children living in "Unsettled" environments may have hyper-regulation of the HPA axis as an adaptation to cope with their environment and this hyper-regulation may induce a depressive mental state. Moreover, our results also demonstrate that children in "Settled" environments have a marked increase in the bedtime salivary OT level compared with TD children. This result suggests that the formation of stable interpersonal relationships with facility staff and their peers may lead to atypical patterns of OT diurnal secretions. Thus, maltreated children living in different environments may differ in their hormonal dysregulation.

\section{Cortisol Studies}

Our results are consistent with previous findings that higher cortisol is associated with depressive problems $(56,57)$. Bernard (13) conducted a similar comparative study of CT patterns in maltreated children who live with their birth parents and those in foster care. However, the results were in complete opposition to ours. This may be because Bernard's study targeted very young children. According to Jessop's (58) review, HPA axis development is not mature in children who are younger than 4 years of age. Prior studies have suggested that maltreated children's CT is unusually low in the morning and slightly high in the evening $(13,19-22)$. However, children with PTSD (24, 59 ) tend to show an elevated or hyperactive pattern. Thus, a consistent picture of maltreated children's CT secretion pattern is currently unavailable. Empirical studies $(18,25)$ have suggested hyperactivity in children who internalize problems. Our "Unsettled" children surely struggle with emotional burdens, unpredictable threats, and a lack of social support. They not only confront the concomitants of CM but also experience numerous stresses within their environments. By contrast, the "Settled" children stay in residential care facilities, protecting them from their prior CM experiences. Our "Settled" children's CT secretion patterns were clearly quite similar pattern to those of TD children. This suggests that the CT reactivity prioritizes

TABLE 2 | Cortisol and Oxytocin measures in maltreated children (unsettled and settled) and typically developing children.

\begin{tabular}{|c|c|c|c|c|c|}
\hline Measures & Unsettled & Settled & TD & Statistics & $p$-value \\
\hline Cortisol AUC (ng/ml)* & $29.96 \pm 15.0$ & $25.41 \pm 8.3$ & $26.08 \pm 9.8$ & $F_{2,58}=0.58$ & ns \\
\hline Oxytocin AUC (pg/ml)* & $385.89 \pm 274.9$ & $784.71 \pm 723.3$ & $835.09 \pm 886.4$ & $F_{2,58}=2.55$ & ns \\
\hline Cortisol slope* & $0.22 \pm 0.2^{a}$ & $0.15 \pm 0.1^{b}$ & $0.12 \pm 0.1^{b}$ & $F_{2,58}=4.16$ & $<0.02$ \\
\hline Oxytocin slope* & $0.11 \pm 1.2$ & $-2.13 \pm 5.9$ & $0.33 \pm 7.3$ & $F_{2,58}=1.00$ & ns \\
\hline
\end{tabular}

Cortisol AUC, cortisol's area under the curve; Oxytocin AUC, oxytocin's area under the curve. ${ }^{a}>^{b}(p<0.05)$.

${ }^{*}$ Covariate items: age, gender, and FSIQ. 
current stress exposure and depressive states, rather than previous early life stresses.

\section{Oxytocin Studies}

A recent study showed an association between exposure to less severe forms of CM and higher OT concentrations, suggesting that adversity may enhance OT secretions, in order to cope with the social environment (60). Although our result was partially consistent with their finding, we only found higher levels of OT at bedtime in the "Settled" CM group. One possible explanation for the higher levels of OT may be related to alteration of the social environment of residential care facilities following the adverse experience.

Previous studies have suggested that OT levels are elevated by social interaction with "Significant others," such as parents (61, 62). In addition, OT levels after social exposure among maltreated children were reported to be higher than those in healthy controls (35). A meta-analysis found that oxytocin administration showed greater attenuation of the cortisol response in laboratory tasks that strongly activated the HPA axis, suggesting that oxytocin may play an important role in HPA dysfunction associated with psychopathology (63). After removal from their homes, "Settled" group children were living in childcare facilities with other children, and without ongoing child maltreatment exposure. These "Settled" children may have adapted to the stable environment in order to survive and thrive, explaining the hyperactivity of OT secretions.

\section{Biological Clocks}

Hormone secretion is regulated by circadian rhythms, which are driven by endogenous biological clocks. A master circadian clock is present in the suprachiasmatic nucleus of the anterior hypothalamus $(64,65)$, which regulates many neuroendocrinological, physiological, and behavioral processes. The alteration in circadian time structure likely plays an important role in the pathogenesis of hormone levels. The present study highlights the need to consider the plight of maltreated children, which is associated with alterations to the HPA axis that affect maltreated children differently in terms of the living environment. CM likely diminishes a person's ability to cope with stress, possibly placing them at risk for psychopathology and ill health. The effect of CM on the circadian rhythm can alter neuroendocrine regulation, produce or diminish HPA axis activity, and change the strength of post-traumatic growth $(12,66,67)$. All these findings suggest that early adversity affects hormonal function, even after several years have elapsed to relieve the stress exposure and to adapt to the social environment.

\section{Study Strengths and Limitations}

A strength of our study is the comprehensive assessment of our participants, by obtaining FSIQ and psychological scale data, and a full trauma evaluation in order to understand CM's developmental influence on CT and OT. The present study indicated lower IQ in maltreated children than in TD children, justifying our use of FSIQ as a covariate in the psychological scale and salivary sample analysis. To our knowledge, this is the first study of basal salivary CT and OT levels comparing CM children in "Unsettled" and "Settled" environments.

Our study has several limitations, the first of which is our modest sample size. Therefore, the relationship between the subsequent environments of maltreated children and their hormonal secretion remains tentative. In the preliminary results of this study, a positive dose-response association between the number of CM types and both hormone levels was not observed. Therefore, the relationship between their levels and abuse history remains unclear. Replication of our result with a larger sample would be worthwhile. Second, we did not control for pubertal influences in the participants. Pubertal transitions (68) are potentially important, because hormonal development is dramatically changed during this period. Previous studies have suggested that child sexual abuse, physical abuse, and a stressful family environment may accelerate sexual maturation $(69,70)$. Third, this was not a longitudinal study and, therefore, resilience was not assessed in our study groups; no information related to outcomes in late adolescence is available for maltreated children. However, the resilience and post-traumatic growth issues of those children must be very important from a physiological, as well as a psychopathological, point of view (71, 72). Fourth, the subjects' socioeconomic status (SES) was not examined in this study because of inadequate information on parents' data among children in residential care facilities. While childhood SES has been associated with neurocognitive and brain development, recent evidence from neurodevelopmental cohort studies has suggested that SES might play an important role in adolescent neuroendocrine development $(73,74)$. Finally, we primarily screened severity of CM using ACE (75), but we did not find any significant differences of environmental factors between CM groups. If we were able to obtain onset and duration of abuse information for those children more precisely, this would better indicate cause and effect relationships within child maltreatment. These limitations should be addressed in the future studies.

\section{CONCLUSION}

Our study revealed dysfunction in the hormonal secretions of CM children living in "Settled" environments and "Unsettled" environments as compared with TD children. Specifically, we found significantly higher awakening CT secretions in children in unstable environments, and significantly higher bedtime OT secretions in children in the residential care facilities. Regarding psychological evaluations, trauma-related depression scores in the "Unsettled" CM group were significantly higher than those in "Settled" CM and TD children. Our study indicates how early adversity influences children's neuroendocrine regulation, physically and psychologically. Longitudinal studies with larger samples are needed to investigate whether an intervention program or treatment is associated with stable changes in hormonal secretions related to stable mental health.

This study did not directly investigate the neural mechanisms underlying any effects. However, hormone release is based on neural function. Combining our experimental paradigm with 
neuroimaging techniques is necessary to elucidate the association between neural function and hormone release. Such paradigms would provide valuable clarification of cause-effect relationships between development and environment.

\section{ACKNOWLEDGMENTS}

We thank Hidemi Akashi for her great contribution to this study; also Yumiko Uryu, Kaori Kobayashi, and Yuki Nakamura, for bridging the clinical and research fields, and special thanks to Dr. Hanako Suzuki for her work.

\section{REFERENCES}

1. Bolhuis JJ, Honey RC. Imprinting, learning and development: from behaviour to brain and back. Trends Neurosci (1998) 21(7):306-11. doi:10.1016/ S0166-2236(98)01258-2

2. Weaver IC, Cervoni N, Champagne FA, D’Alessio AC, Sharma S, Seckl JR, et al. Epigenetic programming by maternal behavior. Nat Neurosci (2004) 7(8):847-54. doi:10.1038/nn1276

3. Fortuin J, van Geel M, Vedder P. Peer influences on internalizing and externalizing problems among adolescents: a longitudinal social network analysis. $J$ Youth Adolesc (2015) 44(4):887-97. doi:10.1007/s10964-014-0168-x

4. Teicher MH, Samson JA. Childhood maltreatment and psychopathology: a case for ecophenotypic variants as clinically and neurobiologically distinct subtypes. Am J Psychiatry (2013) 170(10):1114-33. doi:10.1176/appi. ajp.2013.12070957

5. Rutter M. Developmental psychopathology: a paradigm shift or just a relabeling? Dev Psychopathol (2013) 25(4 Pt 2):1201-13. doi:10.1017/ S0954579413000564

6. De Bellis MD, Keshavan MS, Frustaci K, Shifflett H, Iyengar S, Beers $\mathrm{SR}$, et al. Superior temporal gyrus volumes in maltreated children and adolescents with PTSD. Biol Psychiatry (2002) 51(7):544-52. doi:10.1016/ S0006-3223(01)01374-9

7. Tomoda A, Sheu YS, Rabi K, Suzuki H, Navalta CP, Polcari A, et al. Exposure to parental verbal abuse is associated with increased gray matter volume in superior temporal gyrus. Neuroimage (2011) 54(Suppl 1):S280-6. doi:10.1016/j. neuroimage.2010.05.027

8. Hart H, Rubia K. Neuroimaging of child abuse: a critical review. Front Hum Neurosci (2012) 6:52. doi:10.3389/fnhum.2012.00052

9. Hunter AL, Minnis H, Wilson P. Altered stress responses in children exposed to early adversity: a systematic review of salivary cortisol studies. Stress (2011) 14(6):614-26. doi:10.3109/10253890.2011.577848

10. Pierrehumbert B, Torrisi R, Laufer D, Halfon O, Ansermet F, Beck Popovic M. Oxytocin response to an experimental psychosocial challenge in adults exposed to traumatic experiences during childhood or adolescence. Neuroscience (2010) 166(1):168-77. doi:10.1016/j. neuroscience.2009.12.016

11. Heim C, Bradley B, Mletzko TC, Deveau TC, Musselman DL, Nemeroff CB, et al. Effect of childhood trauma on adult depression and neuroendocrine function: sex-specific moderation by $\mathrm{CRH}$ receptor 1 gene. Front Behav Neurosci (2009) 3:41. doi:10.3389/neuro.08.041.2009

12. van der Vegt EJ, van der Ende J, Kirschbaum C, Verhulst FC, Tiemeier H. Early neglect and abuse predict diurnal cortisol patterns in adults. A study of international adoptees. Psychoneuroendocrinology (2009) 34(5):660-9. doi:10.1016/j.psyneuen.2008.11.004

13. Bernard K, Butzin-Dozier Z, Rittenhouse J, Dozier M. Cortisol production patterns in young children living with birth parents vs children placed in foster care following involvement of Child Protective Services. Arch Pediatr Adolesc Med (2010) 164(5):438-43. doi:10.1001/archpediatrics.2010.54

14. Fries AB, Shirtcliff EA, Pollak SD. Neuroendocrine dysregulation following early social deprivation in children. Dev Psychobiol (2008) 50(6):588-99. doi:10.1002/dev.20319

15. Herman JP, Ostrander MM, Mueller NK, Figueiredo H. Limbic system mechanisms of stress regulation: hypothalamo-pituitary-adrenocortical axis. Prog

\section{FUNDING}

This work was supported by a Grant-in-Aid for Challenging Exploratory Research (Houga) and Scientific Research (C) from the Ministry of Education, Culture, Sports, Science and Technology (MEXT) of Japan (KAKENHI: grant number 25560386 to AT, grant number 15K01753 to TF). Funding organizations had no role in the design or conduct of the study, such as the collection, management, analysis, or interpretation of the data, or the preparation, review, or approval of the manuscript.

Neuropsychopharmacol Biol Psychiatry (2005) 29(8):1201-13. doi:10.1016/j. pnpbp.2005.08.006

16. Schmidt-Reinwald A, Pruessner JC, Hellhammer DH, Federenko I, Rohleder $\mathrm{N}$, Schurmeyer $\mathrm{TH}$, et al. The cortisol response to awakening in relation to different challenge tests and a 12-hour cortisol rhythm. Life Sci (1999) 64(18):1653-60. doi:10.1016/S0024-3205(99)00103-4

17. JohnsonDE, GunnarMRIV.Growthfailureininstitutionalizedchildren.Monogr Soc Res Child Dev (2011) 76(4):92-126. doi:10.1111/j.1540-5834.2011.00629.x

18. Cicchetti D, Rogosch FA, Gunnar MR, Toth SL. The differential impacts of early physical and sexual abuse and internalizing problems on daytime cortisol rhythm in school-aged children. Child Dev (2010) 81(1):252-69. doi:10.1111/j.1467-8624.2009.01393.x

19. Rutter M, Sonuga-Barke EJ, Castle JI. Investigating the impact of early institutional deprivation on development: background and research strategy of the English and Romanian Adoptees (ERA) study. Monogr Soc Res Child Dev (2010) 75(1):1-20. doi:10.1111/j.1540-5834.2010.00548.x

20. Carlson M, Earls F. Psychological and neuroendocrinological sequelae of early social deprivation in institutionalized children in Romania. Ann N Y Acad Sci (1997) 807:419-28. doi:10.1111/j.1749-6632.1997.tb51936.x

21. Dozier M, Manni M, Gordon MK, Peloso E, Gunnar MR, Stovall-McClough KC, et al. Foster children's diurnal production of cortisol: an exploratory study. Child Maltreat (2006) 11(2):189-97. doi:10.1177/1077559505285779

22. Quevedo K, Johnson A, Loman M, Lafavor T, Gunnar M. The confluence of adverse early experience and puberty on the cortisol awakening response. Int J Behav Dev (2012) 36(1):19-28. doi:10.1177/0165025411406860

23. Teicher MH, Andersen SL, Polcari A, Anderson CM, Navalta CP, Kim DM. The neurobiological consequences of early stress and childhood maltreatment. Neurosci Biobehav Rev (2003) 27(1-2):33-44. doi:10.1016/ S0149-7634(03)00007-1

24. De Bellis MD, Baum AS, Birmaher B, Keshavan MS, Eccard CH, Boring AM, et al. A.E. Bennett research award. Developmental traumatology. Part I: biological stress systems. Biol Psychiatry (1999) 45(10):1259-70. doi:10.1016/ S0006-3223(99)00044-X

25. Bruce J, Fisher PA, Pears KC, Levine S. Morning cortisol Levels in preschool-aged foster children: differential effects of maltreatment type. Dev Psychobiol (2009) 51(1):14-23. doi:10.1002/dev.20333

26. Insel TR. The challenge of translation in social neuroscience: a review of oxytocin, vasopressin, and affiliative behavior. Neuron (2010) 65(6):768-79. doi:10.1016/j.neuron.2010.03.005

27. Donaldson ZR, Young LJ. Oxytocin, vasopressin, and the neurogenetics of sociality. Science (2008) 322(5903):900-4. doi:10.1126/science.1158668

28. Feldman R, Gordon I, Zagoory-Sharon O. Maternal and paternal plasma, salivary, and urinary oxytocin and parent-infant synchrony: considering stress and affiliation components of human bonding. Dev Sci (2011) 14(4):752-61. doi:10.1111/j.1467-7687.2010.01021.x

29. Young LJ, Wang Z. The neurobiology of pair bonding. Nat Neurosci (2004) 7(10):1048-54. doi:10.1038/nn1327

30. Ferguson JN, Young LJ, Hearn EF, Matzuk MM, Insel TR, Winslow JT. Social amnesia in mice lacking the oxytocin gene. Nat Genet (2000) 25(3):284-8. doi:10.1038/77040

31. Carter CS. Neuroendocrine perspectives on social attachment and $\begin{array}{lllll}\text { love. Psychoneuroendocrinology (1998) } & \text { 23(8):779-818. doi:10.1016/ }\end{array}$ S0306-4530(98)00055-9 
32. Wismer Fries AB, Ziegler TE, Kurian JR, Jacoris S, Pollak SD. Early experience in humans is associated with changes in neuropeptides critical for regulating social behavior. Proc Natl Acad Sci U S A (2005) 102(47):17237-40. doi:10.1073/pnas.0504767102

33. Ministry of Health LaW. Jido Yougo shisetsu Nyusho jido chohsa Kekka (Institutionalized chidren's investigation result) [Report]. Ministry of Health, Labour and Welfare; 2015 [updated Jan 2015]. Available from: http://www. mhlw.go.jp/file/04-Houdouhappyou-11905000-Koyoukintoujidoukateikyoku-Kateifukushika/0000071184.pdf

34. Suzuki H, Tomoda A. Roles of attachment and self-esteem: impact of early life stress on depressive symptoms among Japanese institutionalized children. BMC Psychiatry (2015) 15:8. doi:10.1186/s12888-015-0385-1

35. Seltzer LJ, Ziegler T, Connolly MJ, Prososki AR, Pollak SD. Stress-induced elevation of oxytocin in maltreated children: evolution, neurodevelopment, and social behavior. Child Dev (2014) 85(2):501-12. doi:10.1111/cdev.12136

36. Sheehan DV, Sheehan KH, Shytle RD, Janavs J, Bannon Y, Rogers JE, et al. Reliability and validity of the mini international neuropsychiatric interview for children and adolescents (MINI-KID). J Clin Psychiatry (2010) 71(3):313-26. doi:10.4088/JCP.09m05305whi

37. Sheehan DV, Lecrubier Y, Sheehan KH, Amorim P, Janavs J, Weiller E, et al. The mini-international neuropsychiatric interview (M.I.N.I.): the development and validation of a structured diagnostic psychiatric interview for DSM-IV and ICD-10. J Clin Psychiatry (1998) 59(Suppl 20):22-33; quiz4.

38. American Psychiatric Association. Diagnostic and Statistical Manual of Mental Disorders, Fourth Edition, Text Revision (DSM-IV-TR). Washington, DC: American Psychiatric Association (2000).

39. Wechsler D. Wechsler Intelligence Scale for Children Fourth Edition USA. San Antonio: Pearson (2003).

40. Follan M, Anderson S, Huline-Dickens S, Lidstone E, Young D, Brown G, et al. Discrimination between attention deficit hyperactivity disorder and reactive attachment disorder in school aged children. Res Dev Disabil (2011) 32(2):520-6. doi:10.1016/j.ridd.2010.12.031

41. Davidson C, O'Hare A, Mactaggart F, Green J, Young D, Gillberg C, et al. Social relationship difficulties in autism and reactive attachment disorder: improving diagnostic validity through structured assessment. Res Dev Disabil (2015) 40C:63-72. doi:10.1016/j.ridd.2015.01.007

42. Dupaul GJ, Power TJ, Anastopoulos AD, Reid R. ADHD Rating Scale-IV, Checklist, Norms, and Clinical Interpretation (Japanese Version). Tokyo: Akashi Shoten (2008).

43. Wakabayashi A, Baron-Cohen S, Uchiyama T, Yoshida Y, Tojo Y, Kuroda $\mathrm{M}$, et al. The autism-spectrum quotient (AQ) children's version in Japan: a cross-cultural comparison. J Autism Dev Disord (2007) 37(3):491-500. doi:10.1007/s10803-006-0181-3

44. DuPaul GJ, Power TJ, Anatopoulos AD, Reid R. ADHD Rating Scale-IV (for Children and Adolescents) Checklists, Norms, and Clinical Interpretation. NY: Guilford Press (1998).

45. Auyeung B, Baron-Cohen S, Wheelwright S, Allison C. The autism spectrum quotient: children's version (AQ-child). J Autism Dev Disord (2008) 38(7):1230-40. doi:10.1007/s10803-007-0504-z

46. Goodman R. The strengths and difficulties questionnaire: a research note. $J$ Child Psychol Psychiatry (1997) 38(5):581-6. doi:10.1111/j.1469-7610.1997. tb01545.x

47. Birleson P, Hudson I, Buchanan DG, Wolff S. Clinical evaluation of a self-rating scale for depressive disorder in childhood (depression self-rating scale). J Child Psychol Psychiatry (1987) 28(1):43-60. doi:10.1111/j.1469-7610.1987. tb00651.x

48. Steinberg AM, Brymer MJ, Kim S, Briggs EC, Ippen CG, Ostrowski SA, et al. Psychometric properties of the UCLA PTSD reaction index: part I. J Trauma Stress (2013) 26(1):1-9. doi:10.1002/jts.21780

49. Asukai N, Kato H, Kawamura N, Kim Y, Yamamoto K, Kishimoto J, et al. Reliability and validity of the Japanese-language version of the impact of event scale-revised (IES-R-J): four studies of different traumatic events. J Nerv Ment Dis (2002) 190(3):175-82. doi:10.1097/00005053-200203000-00006

50. Weiss DS. The Impact of Event Scale-Revised. 2nd ed. New York, NY: The Guilford Press (2004).

51. Lanktree CB, Gilbert AM, Briere J, Taylor N, Chen K, Maida CA, et al. Multiinformant assessment of maltreated children: convergent and discriminant validity of the TSCC and TSCYC. Child Abuse Negl (2008) 32(6):621-5. doi:10.1016/j.chiabu.2007.10.003

52. Fujisawa TX, Tanaka S, Saito DN, Kosaka H, Tomoda A. Visual attention for social information and salivary oxytocin levels in preschool children with autism spectrum disorders: an eye-tracking study. Front Neurosci (2014) 8:295. doi: $10.3389 /$ fnins.2014.00295

53. Dorn LD, Kolko DJ, Susman EJ, Huang B, Stein H, Music E, et al. Salivary gonadal and adrenal hormone differences in boys and girls with and without disruptive behavior disorders: contextual variants. Biol Psychol (2009) 81(1):31-9. doi:10.1016/j.biopsycho.2009.01.004

54. Schwartz EB, Granger DA, Susman EJ, Gunnar MR, Laird B. Assessing salivary cortisol in studies of child development. Child Dev (1998) 69(6):1503-13. doi: $10.2307 / 1132128$

55. Knutsson U, Dahlgren J, Marcus C, Rosberg S, Bronnegard M, Stierna P, et al. Circadian cortisol rhythms in healthy boys and girls: relationship with age, growth, body composition, and pubertal development. J Clin Endocrinol Metab (1997) 82(2):536-40. doi:10.1210/jc.82.2.536

56. Kohrt BA, Hruschka DJ, Kohrt HE, Carrion VG, Waldman ID, Worthman CM. Child abuse, disruptive behavior disorders, depression, and salivary cortisol levels among institutionalized and community-residing boys in Mongolia. Asia Pac Psychiatry (2015) 7(1):7-19. doi:10.1111/appy.12141

57. Dietrich A, Ormel J, Buitelaar JK, Verhulst FC, Hoekstra PJ, Hartman CA. Cortisol in the morning and dimensions of anxiety, depression, and aggression in children from a general population and clinic-referred cohort: an integrated analysis. The TRAILS study. Psychoneuroendocrinology (2013) 38(8):1281-98. doi:10.1016/j.psyneuen.2012.11.013

58. Jessop DS, Turner-Cobb JM. Measurement and meaning of salivary cortisol: a focus on health and disease in children. Stress (2008) 11(1):1-14. doi:10.1080/10253890701365527

59. Carrion VG, Weems CF, Ray RD, Glaser B, Hessl D, Reiss AL. Diurnal salivary cortisol in pediatric posttraumatic stress disorder. Biol Psychiatry (2002) 51(7):575-82. doi:10.1016/S0006-3223(01)01310-5

60. Mizuki R, Fujiwara T. Association of oxytocin level and less severe forms of childhood maltreatment history among healthy Japanese adults involved with child care. Front Behav Neurosci (2015) 9:138. doi:10.3389/ fnbeh.2015.00138

61. Seltzer LJ, Ziegler TE, Pollak SD. Social vocalizations can release oxytocin in humans. Proc Biol Sci (2010) 277(1694):2661-6. doi:10.1098/rspb.2010.0567

62. Tsuji S, Yuhi T, Furuhara K, Ohta S, Shimizu Y, Higashida H. Salivary oxytocin concentrations in seven boys with autism spectrum disorder received massage from their mothers: a pilot study. Front Psychiatry (2015) 6:58. doi:10.3389/ fpsyt.2015.00058

63. Cardoso C, Kingdon D, Ellenbogen MA. A meta-analytic review of the impact of intranasal oxytocin administration on cortisol concentrations during laboratorytasks: moderation by method and mentalhealth. Psychoneuroendocrinology (2014) 49:161-70. doi:10.1016/j.psyneuen.2014.07.014

64. Swaab DF. Development of the human hypothalamus. Neurochem Res (1995) 20(5):509-19. doi:10.1007/BF01694533

65. Dunlap JC. Molecular bases for circadian clocks. Cell (1999) 96(2):271-90. doi:10.1016/S0092-8674(00)80566-8

66. Gonzalez A, Jenkins JM, Steiner M, Fleming AS. The relation between early life adversity, cortisol awakening response and diurnal salivary cortisol levels in postpartum women. Psychoneuroendocrinology (2009) 34(1):76-86. doi:10.1016/j.psyneuen.2008.08.012

67. Nicolson NA, Davis MC, Kruszewski D, Zautra AJ. Childhood maltreatment and diurnal cortisol patterns in women with chronic pain. Psychosom Med (2010) 72(5):471-80. doi:10.1097/PSY.0b013e3181d9a104

68. Rudolph KD, Flynn M. Childhood adversity and youth depression: influence of gender and pubertal status. Dev Psychopathol (2007) 19(2):497-521. doi:10.1017/S0954579407070241

69. Mendle J, Leve LD, Van Ryzin M, Natsuaki MN, Ge X. Associations between early life stress, child maltreatment, and pubertal development among girls in foster care. J Res Adolesc (2011) 21(4):871-80. doi:10.1111/j.1532-7795.2011.00746.x

70. Trickett PK, Noll JG, Putnam FW. The impact of sexual abuse on female development: Lessons from a multigenerational, longitudinal research study. Dev Psychopathol (2011) 23(2):453-76. doi:10.1017/S0954579411000174 
71. Levine SZ, Laufer A, Stein E, Hamama-Raz Y, Solomon Z. Examining the relationship between resilience and posttraumatic growth. J Trauma Stress (2009) 22(4):282-6. doi:10.1002/jts.20409

72. Fujisawa TX, Jung M, Kojima M, Saito DN, Kosaka H, Tomoda A. Neural basis of psychological growth following adverse experiences: a resting-state functional MRI study. PLoS One (2015) 10(8):e0136427. doi:10.1371/journal. pone. 0136427

73. Noble KG, Engelhardt LE, Brito NH, Mack LJ, Nail EJ, Angal J, et al. Socioeconomic disparities in neurocognitive development in the first two years of life. Dev Psychobiol (2015) 57(5):535-51. doi:10.1002/dev.21303

74. Noble KG, Houston SM, Brito NH, Bartsch H, Kan E, Kuperman JM, et al. Family income, parental education and brain structure in children and adolescents. Nat Neurosci (2015) 18(5):773-8. doi:10.1038/nn.3983

75. Felitti VJ, Anda RF, Nordenberg D, Williamson DF, Spitz AM, Edwards $\mathrm{V}$, et al. Relationship of childhood abuse and household dysfunction to many of the leading causes of death in adults. The adverse childhood experiences (ACE) study. Am J Prev Med (1998) 14(4):245-58. doi:10.1016/ S0749-3797(98)00017-8

Conflict of Interest Statement: The authors declare that the research was conducted in the absence of any commercial or financial relationships that could be construed as a potential conflict of interest.

Copyright $\odot 2015$ Mizushima, Fujisawa, Takiguchi, Kumazaki, Tanaka and Tomoda. This is an open-access article distributed under the terms of the Creative Commons Attribution License (CC BY). The use, distribution or reproduction in other forums is permitted, provided the original author(s) or licensor are credited and that the original publication in this journal is cited, in accordance with accepted academic practice. No use, distribution or reproduction is permitted which does not comply with these terms. 